\title{
CONCLUSIONS REACHED AFTER DISCUSSIONS CONCERNING EVAPORATION
}

1 Evaporation is limited by two factors : (a) a supply of water to be evaporated; (b) a supply of energy to provide the heat of vaporization of the water.

2 The extreme condition of high energy supply and low available water introduces great difficulties in the complete physical approach and involves problems of flow of water in unsaturated soils and plant problems of response to moisture stress. These characterize the agricultural problem in the arid zones of the world.

3 At the other extreme of plentiful water it is possible to apply known physical principles with success, leading to the following general principle: At a given time and place there is an upper limit to the rate of evaporation from an extended area of crop, a limit that cannot be increased by addition of more water to the area.

4 The physics of evaporation (water non-limiting) is a complex of energy transfers and transformations, some of the transfers being controlled by aerodynamic factors.

5 Analysis of the complex shows that both the energy exchanges and the aerodynamic transfers are essentially meteorological in character, leading to another general principle:

The maximum rate of evaporation (as defined in par. 3) is determined primarily by weather.

6 With certain restrictions, this maximum rate appears to be largely independent of the kind of crop or the kind of soil on which it is growing. Under these conditions it is useful to give the maximum rate a special name viz. potential transpiration (or potential evapotranspiration), which may be defined in the following manner:

The potential transpiration rate is the rate of evaporation from an extended surface of short green crop ${ }^{1}$ ) actively growing ${ }^{2}$ ), completely shading the ground, of uniform height and not short of water.

7 The concept of potential transpiration is useful even in qualitative evaluation of evaporation.

8 There are alternative ways of trying to obtain quantitative values of potential transpiration. All make use of meteorological data. The most satisfactory incorporate both energy and aerodynamic aspects of the evaporation process.

9 Physical uncertainties in the estimation of potential transpiration arise from two sources :

(a) incomplete knowledge of the physics of

(i) the radiant energy exchanges that take place in the open and under glass; and

(ii) the turbulent transfer processes in the atmosphere.

(b) the difficulty of measuring the relevant physical quantities.

1) At the present stage of investigation and research it is impossible to give a precise definition of what is implied by the word "short green crop". The word "short" applies to crops common to agriculture and horticulture.

2) "Actively growing" implies that the crop is not or has not been recently subject to a growth check, such as might arise from severe night frost, or close cutting of a forage crop. 
10 Biological uncertainties arise from many sources, including the morphology of the species, the physiological stage and activity of the plants. Although some of the problems are obviously physical in character it is clear that the cooperation of the biologist is essential in attempting to resolve present difficulties.

11 In spite of the defects listed in paras. 9 and 10 it is clear that evaporation in agriculture (water non-limiting) is fundamentally a physical phenomenon. For many practical purposes the rate can be regarded as dictated by meteorological factors and can be expressed quantitatively if values of the relevant weather factors are known.

12 With precise instruments and a high degree of skill in using them, it is possible, after somewhat complicated calculations, to evaluate rates of actual evaporation. This is work of fundamental importance in meteorological physics, the results of which cannot fail to benefit agricultural science and ultimately agricultural practice.

13 At present, however, difficulties are experienced in applying such results obtained over small intervals of time to intervals of agricultural or hydrological significance. Meanwhile, as a guide to present action in the field, it is necessary to accept something less than perfection and adopt a method that will make use of measurements more easily obtainable, in calculations of a simpler nature. There exists a wide range of practical problems in which an approximate answer is acceptable.

14 Such methods are available but are not easy to check, because of the difficulty in drawing up an accurate water balance sheet under field conditions. However, there are sufficient observations on a field scale to indicate that evaporation estimates obtained in this manner can be applied to problems of irrigation development and practice, especially when the irrigation is intended to maintain non-limiting water conditions. The possibility of making an estimate of potential transpiration depends on the availability of meteorological data, and the accuracy of the estimate will be limited as indicated in paras. 9 and 10. At present it is risky to apply the existing approximate formulae to make estimates of water loss over short periods of time.

15 Calculations of evaporation from large open water surface seem to be reasonably accurate.

16 Application to the water balance in areas with inadequate rainfall demands assumptions about the differences between potential and actual water loss. The results are subject to a considerable uncertainty even in humid areas; where, nevertheless, useful results have been obtained.

17 These assumptions are needed because knowledge of the rate of transmission of water through the soil and the plant is insufficient, and will remain so until further biological and physical research is carried out. Existing techniques for measuring soil water are inadequate for this research, so imposing a serious limitation to the possible rate of immediate advance. Increased efficiency in such measurements is urgently required.

18 It is essential that there should be close collaboration between research workers in physics, hydrology, biology and agronomy. Such collaboration should be international and should be effective in all stages in the research. For example, on the instrumental side it is very desirable that research workers in different countries should be able to exchange experience of failures as well 
as of successes. It is not always satisfactory to depend on access to published papers; there should be facilities for personal consultations of a confidential nature both before and during experiments. This can save much time and money as critical assessments cannot always be made by reference to the literature.

19 In conclusion we affirm two beliefs :

First : that although the importance of water in man's environment is everywhere recognized, the research effort designed to enable water to be controlled is not commensurate with that importance.

Second : that the discovery of the facts related to the laws of water movement is the proper role of the physicist in this field of work.

20 Research and its application must progress side by side, recognising that it is neither necessary nor desirable to wait for scientific perfection before offering practical help. The physicist's dual task is clear : to improve his own knowledge and to intensify his help where it is needed. 